\title{
Distributed Predictive Secondary Control for Voltage Restoration and Economic Dispatch of Generation for DC Microgrids
}

\author{
Alex Navas-Fonseca \\ Electrical Engineering \\ University of Chile \\ Santiago, Chile \\ alex.navas@ug.uchile.cl claudio.burgosmellado1@nottingham.ac.uk
}

\author{
Enrique Espina \\ Electrical Engineering \\ University of Santiago of Chile \\ Santiago, Chile \\ enrique.espinag@usach.cl
}

\author{
Erwin Rute \\ Electrical Engineering \\ University of Chile \\ Santiago, Chile \\ erwin.rute@gmail.com
}

\author{
Juan S. Gómez \\ Electrical Engineering \\ Pontificia Universidad Católica de Chile \\ Santiago, Chile \\ jugomez@ug.uchile.cl
}

\author{
Doris Sáez \\ Electrical Engineering \\ University of Chile \\ Santiago, Chile \\ dsaez@ing.uchile.cl
}

\author{
Mark Sumner \\ Electrical and Electronic Engineering \\ University of Nottingham \\ Nottingham, United Kingdom \\ Mark.Sumner@nottingham.ac.uk
}

\begin{abstract}
DC microgrids ease the integration of renewables and can be easily put together with plug-and-play operation. However, most control techniques in DC microgrids focus on voltage regulation without considering its economical operation. Furthermore, the economic dispatch of different generation is usually implemented at a higher control level for long-term operation, and this is subject to prediction uncertainties. Therefore, this paper proposes a novel distributed model predictive control strategy for economic dispatch of the system generation and voltage restoration at the same time-scale in DC microgrids. Each distributed generator achieves both objectives using local measurements and predicted information from neighboring units. Experimental results in a $6 \mathrm{~kW}$ testbed validate the distributed structure of the predictive controller for communication problems and in the presence of plug-and-play scenarios without modifying and/or adjusting the control system parameters.
\end{abstract}

Index Terms-Distributed predictive control, predictive optimal dispatch, Microgrids, cooperative control, voltage regulation.

\section{INTRODUCTION}

Microgrids (MGs) are the main enablers for integrating renewable resources in modern power systems. MGs can be classified as AC MGs, hybrid AC/DC MGs, and DC MGs. Modern distributed generators (DGs) have a DC nature which can reduce conversion stages and improve its efficiency [1]. DC MGs are efficient, reliable, and ease the integration of renewable energies [1]-[3].

This study was supported by FONDEQUIP EQM130158, Instituto Sistemas Complejos de Ingeniería (ISCI) ANID PIA/BASAL AFB180003, Solar Energy Research Center SERC-Chile ANID/FONDAP/15110019, The Department of Electrical Engineering of the University of Chile, and PhD scholarships from ANID- PCHA/Doctorado Nacional/2017-21171858, ANID/Doctorado Nacional 2019-21190961 and from Secretaría de Educación Superior, Ciencia, Tecnología e Innovación de Ecuador, SENESCYT/ARSEQBEC-005848-2018.
Focusing on isolated DC MGs, its control system is usually divided into a three-level centralized hierarchical structure. The control levels are primary, secondary, and tertiary, where each control level has a different purpose [1]. All control levels have a different time-scale, where the primary control level is the fastest, and it distributes load power among the DGs without considering economic aspects. The secondary level regulates the MG's voltage and active power sharing [4]-[6], and the tertiary level manages the MG's economic operation. However, this hierarchical control structure is inherited from large-scale power systems [4]. In contrast, as MGs are based on non-dispatchable renewables with power electronic interfaces, they may be susceptible to fast changes in generation and consumption. Thus, a slow economic dispatch may be inappropriate [7]. Furthermore, centralized control techniques have a common point of failure in the communication network, present a high computational burden, and plug-and-play operation of DG units is difficult to achieve [1], [8].

For these reasons, distributed control techniques using consensus strategies are the current trend at the secondary level [8]. Algorithms based on a consensus strategy over the incremental costs are a prominent control technique for distributed economic dispatch of generation [7], [9]-[14]. Despite these issues, to the best of the authors' knowledge, distributed model predictive control (DMPC) has not been studied thoroughly at the secondary level for DC MGs. This paper proposes a secondary DMPC technique for economic dispatch of DGs and voltage restoration in DC MGs. The proposed DMPC scheme is experimentally validated in a multi-nodal DC MG with three DGs, where each DG is a controllable generator with its own physical and cost parameters. The contributions of this paper are:

i) A DMPC scheme is proposed for optimal dispatch of 


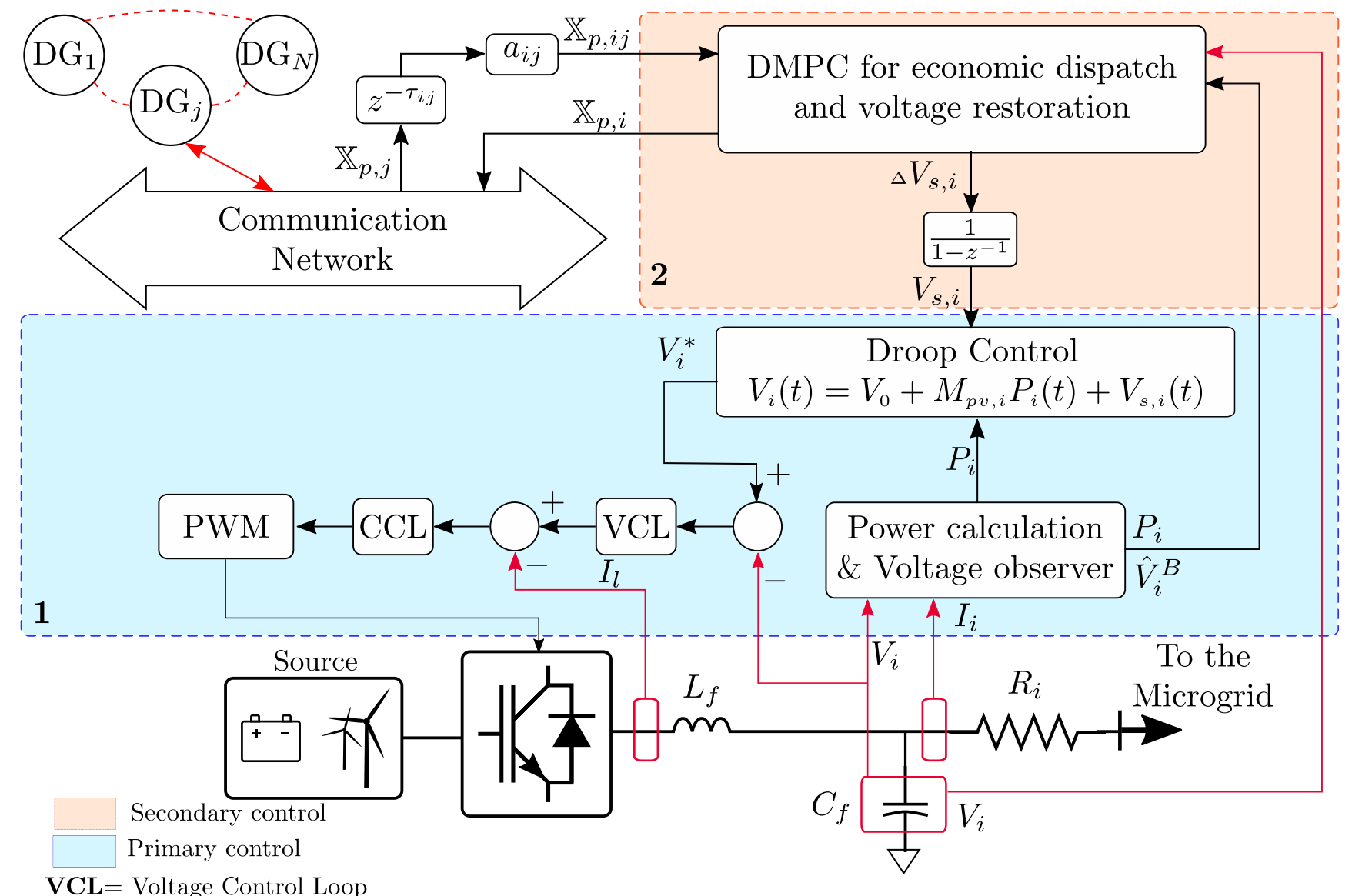

$\mathbf{V C L}=$ Voltage Control Loop

$\mathbf{C C L}=$ Current Control Loop

Fig. 1. General control diagram of $\mathrm{DG}_{i}$.

DGs and voltage restoration within the same controller at the secondary control level.

ii) The DMPC includes dynamic models as equality constraints to predict the DGs' behavior. Furthermore, physical constraints are also included.

iii) The DMPC does not require the modeling of the full MG and uses only the usual local measurements.

The rest of this paper is organized as follows. In Section II, the theoretical background and the proposed DMPC controller are explained in detail. Section III presents the experimental setup and the experimental results obtained. Finally, the conclusions are presented in Section IV.

\section{Proposed Distributed Predictive Control}

\section{A. Proposed Distributed Control Scheme}

Consider a DC MG with a set of $\mathbb{N}$ DGs with $\mathbb{N}=$ $\{1, \ldots, N\}$ that share information among them to achieve cooperative objectives. The information is shared through a full-duplex communication network, which suffers from communication delays. The communication delays are represented by $\tau_{i j}$ and are expressed in sampling periods $\left(\tau_{i j}=\tau_{j i}\right)$ while the communication topology between the DGs is defined by the terms $a_{i j}$. The bidirectional connectivity from the ith DG
$\left(D G_{i}\right)$ to $D G_{j}$ and vice-versa is defined by the adjacency term $a_{i j}$ (1) of the adjacency matrix $A$ (defined in Section III) [15].

$$
a_{i j}(k)= \begin{cases}1 & \text { Data from } \mathrm{DG}_{j} \text { arrives to } \mathrm{DG}_{i} \text { at instant } k \\ 0 & \text { Data from } \mathrm{DG}_{j} \text { does not arrive to } \mathrm{DG}_{i} \text { at instant } k \\ 0 & j=i\end{cases}
$$

The following description and mathematical analysis are carried out for $D G_{i}$, and for the rest of DGs, the analysis is analogous. It has been demonstrated that the economic dispatch of the DG can be achieved in a distributed fashion through a consensus on the incremental cost (IC) [7], [13], [14] (a detailed explanation can be found in [13]). The IC for $D G_{i}$ can be defined as $\eta_{i}\left(P_{i}\right)=2 a_{i} P_{i}+b_{i}$, where $P_{i}$ is the active power contribution of $D G_{i}, a_{i}$ and $b_{i}$ are cost parameters defined in Section III. The economic dispatch is achieved when all DGs reach a common IC, i.e. $\eta_{i}=\eta_{j}$, where $\eta_{j}$ is the IC of neighboring DGs. Note that the supplydemand balance is met when the consensus is achieved. In this paper, a new distributed cooperative predictive scheme for DC MGs is formulated based on the IC, while the voltage on the MG is regulated at the same time. It is based on distributed model predictive control (DMPC). Note that the proposed controller does not require the modeling of the $\mathrm{MG}$ electrical topology. Conversely, it only needs the usual measurements at 
the primary control level and information from communicated neighboring DG units.

The control structure for the $D G_{i}$ unit is shown in Fig. 1 . As seen, each DG is configured as a voltage source converter and has an LC filter at its output. Two control levels can be distinguished, i.e., primary and secondary control levels. The primary control level (blue box) is made up of a $V-P$ droop controller, outer voltage controller, and inner current controller. The predictive controller for both the economic dispatch and voltage regulation is implemented at the secondary level (orange box). This controller receives as inputs the local measurements/estimates $\left(P_{i}(k), V_{i}(k) \hat{V}_{i}^{B}(k)\right)$ of the $D G_{i}$ unit and the results of the optimization problems of communicated neighboring DG units. The controller has two outputs, which are the control actions sequence (vector $\Delta V_{s, i}$ ) and the results of the local optimization problem $\mathbb{X}_{p, i}$ (predicted values), both defined in Section II-B. The former passes through a discrete integrator to guarantee zero error in steady-state, and the latter is sent to the neighboring DG units via the communication network.

The dynamic models (2) and (3) represent the behavior of the DGs included in the DMPC formulation. In particular, the droop controller (2) allows that a change in active power be perceived as a change in voltage. $V_{i}$ is the output voltage, $P_{i}$ is the active power transferred to the MG, calculated by (3), $V_{0}$ is the nominal voltage and $V_{s, i}$ is the secondary control action. Equation (3) represents the power contribution of $D G_{i}$ to the MG. Note that, by using (3), a complete power flow electrical model of the MG is not needed. Instead, only the voltage $\hat{V}_{i}^{B}$ after the coupling resistor $R_{i}$ (with $G_{i}=1 / R_{i}$ ) is used, and it is computed using Ohm's law.

$$
\begin{gathered}
V_{i}(t)=V_{0}+M_{i} P_{i}(t)+V_{s, i}(t) \\
P_{i}(t)=V_{i}(t) \cdot I_{i}(t)=G_{i} V_{i}(t)\left(V_{i}(t)-\hat{V}_{i}^{B}(t)\right)
\end{gathered}
$$

\section{B. Distributed Predictive Control Formulation}

The objectives of the proposed controller are to guarantee the economic dispatch, i.e., the MG load is supplied by the most economical units, as long as their power capacity is not exceeded, and to restore the average voltage to its nominal value.

This controller uses a discretized predictive version of the previous defined models (2) and (3). The models (2) and (3) are discretized and generalized for the prediction horizon $\left(N_{y}\right)$, where $k=n T_{s e c}, n \in \mathbb{Z}^{+}$, and $T_{s e c}$ is the controller sample time. The incremental operator $(\Delta f(k)=[f(k)-f(k-1)])$ is applied in (2) to formulate the optimization problem in function of the control action variation $\left(\Delta V_{s, i}\right)$. Furthermore, (3) is linearized using the forward Euler method around the measured/estimated point $\left\{V_{i}(k), \hat{V}_{i}^{B}(k), P_{i}(k)\right\}$. Then, both models are generalized for $k+m$ steps ahead, where $m \in \mathbb{Z}^{+}$. The resulting predictive models of (2) and (3) are given by (4) and (5), respectively.

$$
\begin{aligned}
V_{i}(k+m)= & M_{i}\left[P_{i}(k+m)-P_{i}(k+m-1)\right] \\
& +V_{i}(k+m-1)+{ }_{\Delta} V_{s, i}(k+m-1)
\end{aligned}
$$

$$
\begin{aligned}
P_{i}(k+m)= & {\left[V_{i}(k+m)-V_{i}(k)\right] G_{i}\left[2 V_{i}(k)-\hat{V}_{i}^{R}(k)\right] } \\
& +P_{i}(k)
\end{aligned}
$$

The predictive model of the incremental cost (IC), which is defined in (6), is included in the formulation to obtain the most economic dispatch of generation. A local approximation of the average voltage is presented in (7), which depends on the communication terms $a_{i j}(k)$ and the estimated delay $\left(\hat{\tau}_{i j}\right)$, defined as one sample period on the secondary level. Inequality constraints to limit the active power contribution (8), according to their physical capacities, and the local DG output voltage (9) are included to keep the DGs voltages within acceptable ranges and bound the solution space [16].

$$
\begin{gathered}
\eta_{i}(k+m)=2 a_{i} P_{i}(k+m)+b_{i} \\
\bar{V}_{i}(k+m)=\frac{V_{i}(k+m)+\sum_{j=1}^{N} a_{i j}(k) V_{j}\left(k+m-\hat{\tau}_{i j}\right)}{1+\sum_{j=1}^{N} a_{i j}(k)} \\
P_{i, \min } \leq P_{i}(k+m) \leq P_{i, \max } \\
V_{i, \min } \leq V_{i}(k+m) \leq V_{i, \max }
\end{gathered}
$$

The cost function (10) comprises three quadratic terms. The first term achieves a consensus over the predicted incremental costs. The second term regulates the average MG voltage, and the third term penalizes the secondary control action variation to obtain a smooth response. The terms $\lambda_{1 i}, \lambda_{2 i}$ and $\lambda_{3 i}$ are the tuning parameters. Note that the consensus on the IC depends on the communication terms $a_{i j}(k)$ and the estimated delay $\left(\hat{\tau}_{i j}\right)$. The optimization problem composed of the constraints (4)-(9) and cost function (10) is synthesized as a Quadratic Programming problem with linear constraints. The optimization output is composed of the predictive vector $\mathbb{X}_{p, i}$ and the future control sequence $\mathbb{X}_{\Delta, i}$ presented in (11) and (12), respectively.

$$
\begin{array}{r}
J_{i}(k)=\sum_{j=1, j \neq i}^{N} \sum_{m=1}^{N_{y}} \lambda_{1 i} a_{i j}(k)\left(\eta_{i}(k+m)-\eta_{j}\left(k+m-\hat{\tau}_{i j}\right)\right)^{2} \\
+\sum_{k=1}^{N_{y}} \lambda_{2 i}\left(\bar{V}_{i}(k+m)-V_{0}\right)^{2}+\sum_{k=1}^{N_{u}} \lambda_{3 i}\left(\Delta V_{s, i}(k+m-1)\right)^{2}
\end{array}
$$

$\mathbb{X}_{p, i}=\left\{\bar{V}_{i}(k+m), V_{i}(k+m), P_{i}(k+m), \eta_{i}(k+m)\right\}_{k=1}^{N_{y}}$

$$
\mathbb{X}_{\Delta, i}=\left\{\Delta V_{s, i}(k+m-1)\right\}_{k=1}^{N_{u}}
$$

\section{EXPERIMENTAL SETUP AND RESUlts}

\section{A. Experimental DC Microgrid Configuration}

The performance of the proposed DMPC scheme was evaluated in a case study using the experimental DC MG topology depicted in Fig. 2.a. The experimental DC MG was implemented in the MG Control Lab of the University of Chile [17]. It is composed of three DG units, which are emulated by the PM15I6F06 Triphase ${ }^{\circledR}$ units, as illustrated in Fig. 2.b. Each DG unit has a real-time target (RTT) computer, in which 


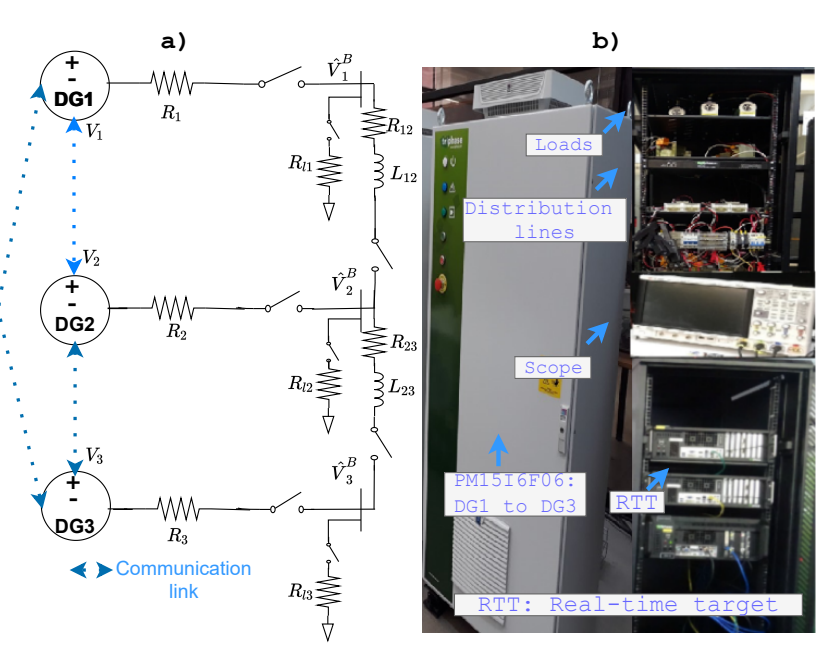

Fig. 2. MG topology for the validation of the DMPC scheme.

TABLE I

MG PARAMETERS AND LOADS

\begin{tabular}{|l|l|l|}
\hline \multicolumn{3}{|c|}{ MG parameters } \\
\hline Parameter & Description & Value \\
\hline$T_{\text {prim }}[\mathrm{s}]$ & Primary level sample period & $1 /\left(16 \cdot 10^{3}\right)$ \\
\hline$R_{l 1}[k W]$ & Load 1 & 0.86 \\
\hline$R_{l 2}[k W]$ & Load 2 & 0.56 \\
\hline$R_{l 3}[k W]$ & Load 3 & 0.82 \\
\hline$R_{i}[\Omega]$ & Coupling resistor & 0.47 \\
\hline$R_{12} ; R_{23}[\Omega]$ & Distribution line resistors & $0.75 ; 0.50$ \\
\hline$L_{12} ; L_{23}[\mathrm{mH}]$ & Distribution line inductors & 2.5 \\
\hline$V_{0}[\mathrm{~V}]$ & Nominal voltage & 100 \\
\hline
\end{tabular}

TABLE II

DGS PARAMETERS

\begin{tabular}{|l|l|l|l|}
\hline Parameter & DG1 & DG2 & DG3 \\
\hline $\mathrm{a}\left[\$ / k W h^{2}\right]$ & 0.264 & 0.444 & 0.5 \\
\hline $\mathrm{b}[\$ / k W h]$ & 0.067 & 0.111 & 0.125 \\
\hline $\begin{array}{l}\text { Maximum power } \\
\text { capacity }\left(P_{i, \max }\right)[\mathrm{kW}]\end{array}$ & \multicolumn{3}{|c|}{2} \\
\hline $\begin{array}{l}V-P \text { droop } \\
\text { coefficient }\left(M_{i}\right)\left[\frac{V}{W}\right]\end{array}$ & \multicolumn{4}{|c|}{$-5 \cdot 10^{-3}$} \\
\hline
\end{tabular}

TABLE III

CONTROLLER PARAMETERS AND WEIGHTS

\begin{tabular}{|l|l|l|}
\hline Parameter & Description & Value \\
\hline$T_{s e c}[\mathrm{~s}]$ & Secondary controller sample time & 0.05 \\
\hline$\hat{\tau}_{i j}[\mathrm{~s}]$ & Estimated communication delay & 0.05 \\
\hline$N y$ & Prediction horizon & 5 \\
\hline$N u$ & Control horizon & 5 \\
\hline$\left[V_{\max }, V_{\min }\right][V]$ & Voltage limits & {$[95,105]$} \\
\hline$\lambda_{1 i}\left[(1 / W)^{2}\right]$ & Active power dispatch weighting factor & $1 \cdot 10^{-3}$ \\
\hline$\lambda_{2 i}\left[(1 / V)^{2}\right]$ & Average voltage error weighting factor & $7 \cdot 10^{1}$ \\
\hline$\lambda_{3 i}\left[(1 / V)^{2}\right]$ & Control action variation weighting factor & $8 \cdot 10^{2}$ \\
\hline
\end{tabular}
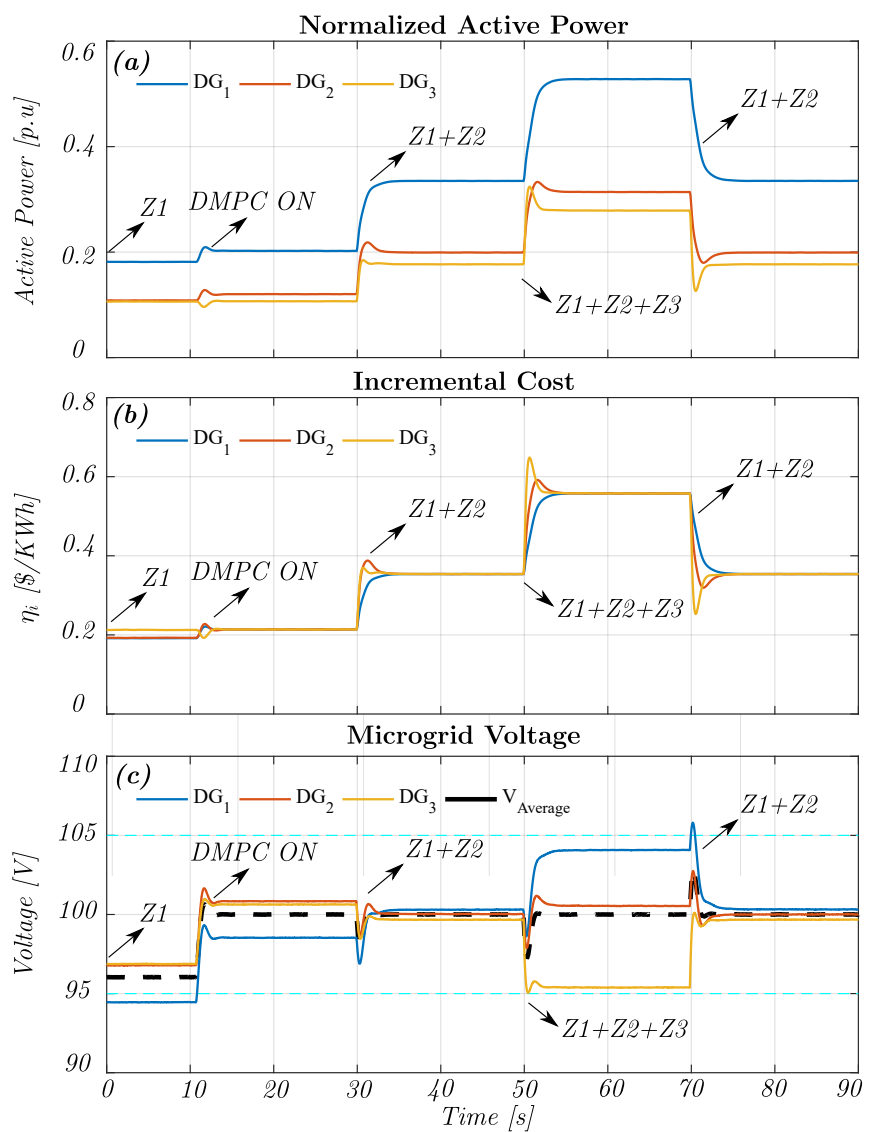

Fig. 3. Load impact test, a) Active power injection, b) incremental cost consensus, b) Voltage regulation

the DMPC control algorithm shown in Fig. 1 is implemented. Table I and Table II present the MG electrical parameters, DG physical parameters and the DG cost parameters, respectively. The cost parameters were obtained from [7].

The DMPC parameters and weighting factors are presented in Table III. The prediction and control horizons were selected as 10 steps ahead. With these values, the controller has a good performance, and both the computational burden and the traffic over the communication network are reduced. Moreover, the weighting factors were tuned heuristically, looking for a tradeoff between the control objectives [8], [13].

The DMPC strategy was validated under three scenarios in the experimental DC MG. The first scenario highlights the DMPC performance when the MG is subject to load changes. The second scenario validates the Plug-and-Play capability, where DG1 is disconnected and reconnected to the DC-MG. The last scenario validates the resilience of the controller when there are communication delays over the communication network.

\section{B. Scenario I (Base Case) - Load Changes}

This scenario presents the behavior of the DMPC scheme when there are load variations in the MG. The communication network is described by the adjacency matrix $\mathrm{A}$ in (13). The test starts with the primary control enabled (see the blue box in Fig. 1) and $R_{l 1}$ connected. The results are presented in 

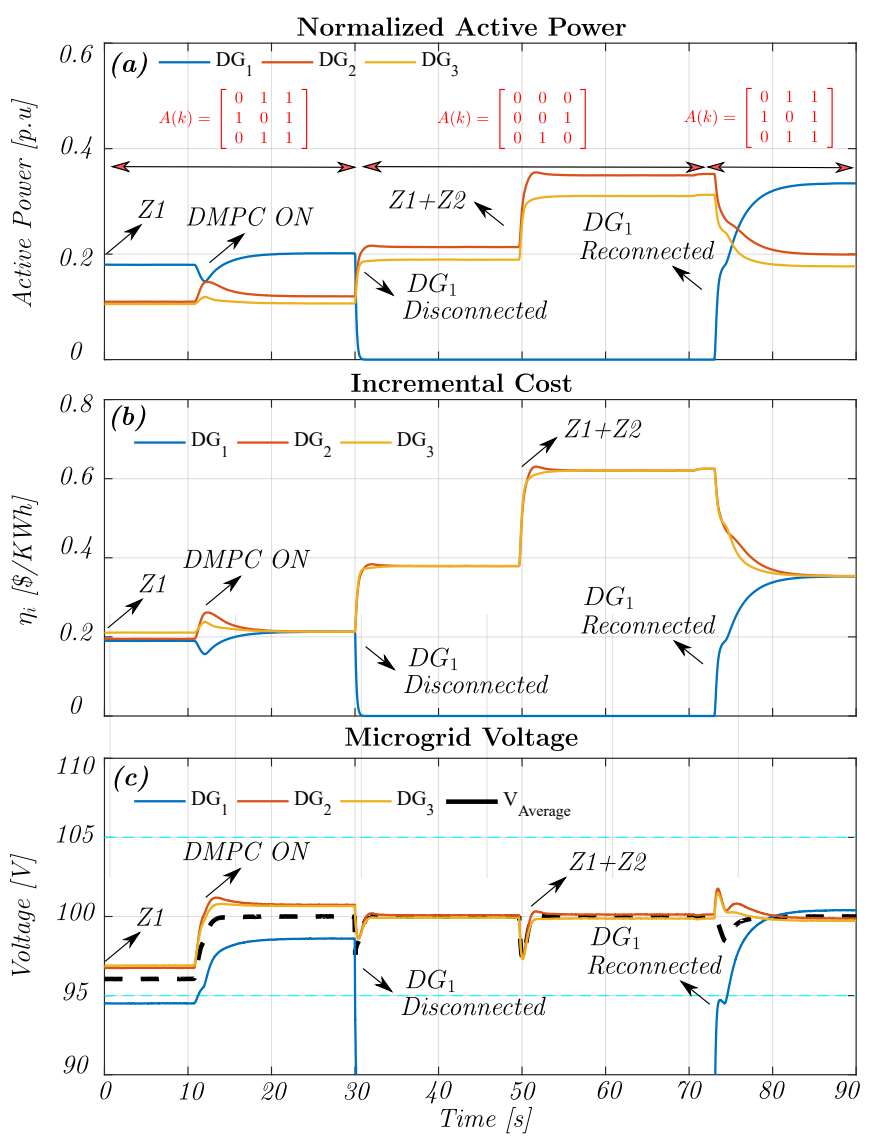

Fig. 4. Plug-and-Play test, a) Active power injection, b) incremental cost consensus, c) Voltage regulation

Fig. 3. Note that as the DMPC is not enabled from $t=0 s$ to $t=10 \mathrm{~s}$, the droop control shares the active power among the DG units according to their droop slopes without considering generation costs. Also, as seen in Fig. 3.b and Fig. 3.c before $t=10 \mathrm{~s}$, there is not a consensus among incremental costs and the MG average voltage deviates from its nominal value.

$$
A(k)=\left[\begin{array}{lll}
a_{11} & a_{12} & a_{13} \\
a_{21} & a_{22} & a_{23} \\
a_{31} & a_{32} & a_{33}
\end{array}\right]=\left[\begin{array}{lll}
0 & 1 & 1 \\
1 & 0 & 1 \\
1 & 1 & 0
\end{array}\right]
$$

At $t=10 \mathrm{~s}$, the proposed DMPC controller is enabled; thus, the active power is redistributed, as observed in Fig. 3.a. The DMPC redispaches the active power according to the DG's operating costs, where DG1 is the least expensive, DG2 has an intermediate cost, and DG3 is the most expensive. This redispatch is achieved by the consensus in the incremental cost depicted in Fig. 3.b $(t=10 s$ and onwards), which guarantees the economic operation of the MG. Furthermore, the microgrid average voltage is restored to its nominal value, as shown in Fig. 3.c. Note that the voltages at the DGs' outputs are kept within the limits presented in Table III (cyan dashed lines); this is because constraint (9) limits the maximum voltage deviation at the output of each DG.

Finally, at 30 and 50 seconds, loads $R_{l 2}$ and $R_{l 3}$ are connected, respectively. Additionally, load $R_{l 3}$ is disconnected at $t=70$ (see Fig. 2.a). The results of Fig. 3 show that during all the load perturbations the controller presents a good dynamic response with negligible overshoots and with settling times below 3 seconds.

\section{Scenario II - Plug-and-Play}

This test presents the DMPC performance when unexpectedly DG1 fails, i.e., it is disconnected from the DC MG. The test starts with all DGs operating without the DMPC and $R_{l 1}$ connected (see Fig. 4 before $t=10 \mathrm{~s}$ ). The DMPC is enabled at $t=10 \mathrm{~s}$, and its initial adjacency matrix is represented by (13). At $t=30 \mathrm{~s}$ DG1 is disconnected from both the electrical system and the communication network; thus, the MG continues operating with the remaining units, and the adjacency matrix is modified, as shown at the top of Fig. 4.a. Load $R_{l 2}$ is connected at $t=50 \mathrm{~s}$, and DG1 is reconnected at $t=70 \mathrm{~s}$. It must be noted that in this test, the proposed controller autonomously identifies when DG1 is disconnected and reconnected by updating the consensus terms in (7) and (10) only with the received information.

The results in Fig. 4 show that when DG1 is disconnected, the remaining DGs increase their power contribution respecting economic dispatch, i.e., DG2 takes more load than DG3 (see Fig. 4.a). Furthermore, the remaining DGs achieve consensus on the incremental cost (see Fig. 4.b), and the average voltage is restored to its nominal value (see Fig. 4.c). Therefore, during all the events, the DMPC objectives are achieved, and operational constraints are respected, showing the effectiveness of the proposed DMPC control scheme.

\section{Scenario III - Communication Delay}

This test evaluates the resilience of the controller when there is a constant delay $\tau_{i j}$ in the entire communication network, while the estimated delay $\hat{\tau}_{i j}$ is kept constant as one sample period. The delays considered for this test are $\tau_{i j}=0.5 \mathrm{~s}$ and $\tau_{i j}=1 \mathrm{~s}$. These delays are considered as medium and large delays for the secondary control level (see [7], [14]). Note that the last delay is equivalent to 20 sample periods, which is two times the prediction horizon $N_{y}$. For this test, the MG is subject to the same load perturbations of scenario I, and the adjacency matrix, represented by (13), is kept constant for the whole test. The results are presented in Fig. 5.

Fig. 5.a and Fig. 5.b present the active power behavior for the medium and large delays, respectively. In the same way, Fig. 5.c and Fig. 5.d present the voltage restoration performance for the medium and large delays. It is observed that the active power is practically not affected by the delay. Even under the large delay $\left(\tau_{i j}=1 s\right)$, the settling time is below five seconds, and the increase in the overshoot is negligible. Furthermore, the voltage restoration objective is the most affected variable when there are large delays in the communication network. It is observed that the delay affects its overshoot and settling time. Nevertheless, for the worstcase scenario $\left(\tau_{i j}=1 s\right)$, its overshoot is less than $0.3 \%$ and its settling time is below ten seconds.

The results demonstrate that the controller can adequately handle delays. This is because the DMPC strategy posses the 

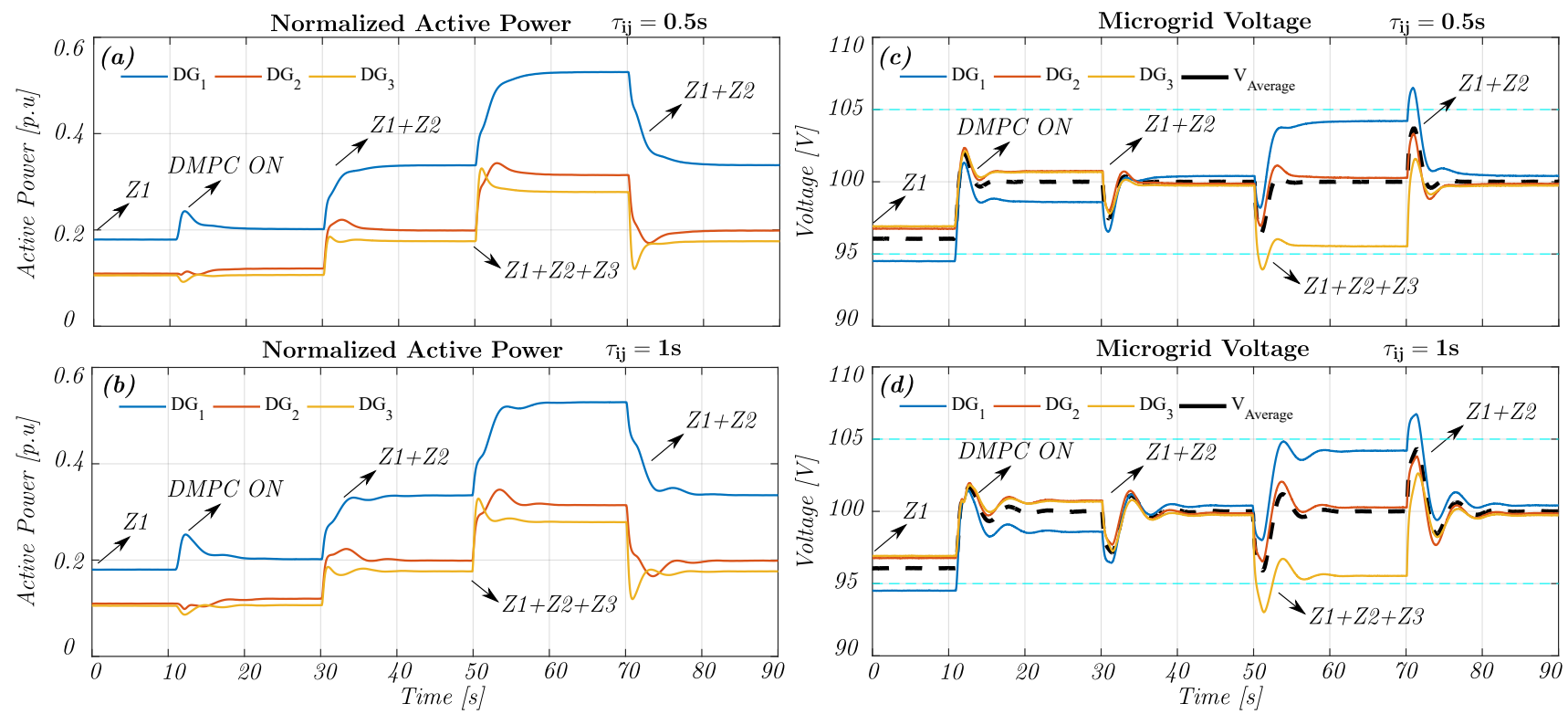

Fig. 5. Communication delay test, a) Normalized active power consensus for $\tau_{i j}=0.5 \mathrm{~s}$, b) Normalized active power consensus for $\tau_{i j}=1 \mathrm{~s}$, c) Voltage regulation for $\left.\tau_{i j}=0.5 \mathrm{~s}, \mathrm{~d}\right)$ Voltage regulation for $\tau_{i j}=1 \mathrm{~s}$. The dashed cyan lines represent the predefined band limits for voltage regulation.

rolling horizon property, which compensates for delays [18] by determining the adequate control action sequence at each sample time, even with past information from neighboring DGs.

\section{CONCLUSION}

This paper presented a novel DMPC strategy to tackle optimal dispatch and voltage regulation for DC MGs without timescale separation. The proposed controller can achieve both objectives while respecting operating constraints. Three experimental scenarios validate the dynamic performance of the DMPC scheme. The proposed controller has good performance under communication delays and the connection/reconnection of DGs. Future research will be focused on the extension of the control strategy for hybrid AC/DC MGs.

\section{REFERENCES}

[1] S. K. Sahoo, A. K. Sinha, and N. K. Kishore, "Control techniques in AC, DC, and hybrid AC-DC microgrid: A Review," IEEE Trans. Emerg. Sel. Topics Power Electron., vol. 6, no. 2, pp. 738-759, Dec. 2018.

[2] T. Dragicevic, X. Lu, J. C. Vasquez, and J. M. Guerrero, "DC Microgrids - Part I: A Review of Control Strategies and Stabilization Techniques," IEEE Trans. Power Electron., vol. 31, no. 7, pp. 4876-4891, Jul. 2016.

[3] T. Dragičević, X. Lu, J. C. Vasquez, and J. M. Guerrero, "DC Microgrids - Part II: A Review of Power Architectures, Applications, and Standardization Issues," IEEE Trans. Power Electron., vol. 31, no. 5, pp. 3528-3549, May 2016.

[4] J. M. Guerrero, J. C. Vásquez, J. Matas, L. G. de Vicuna, and M. Castilla, "Hierarchical control of droop-controlled ac and dc microgrids-a general approach toward standardization," IEEE Trans. Ind. Electron., vol. 58, no. 1, pp. 158-172, Jan. 2011.

[5] S. Anand, B. G. Fernandes, and J. M. Guerrero, "Distributed control to ensure proportional load sharing and improve voltage regulation in lowvoltage DC microgrids," IEEE Trans. Power Electron., vol. 28, no. 4, pp. 1900-1913, Apr. 2013.

[6] S. Sahoo and S. Mishra, "A Distributed Finite-Time Secondary Average Voltage Regulation and Current Sharing Controller for DC Microgrids," IEEE Trans. Smart Grid, vol. 10, no. 1, pp. 282-292, Jan. 2019.
[7] J. Llanos, D. E. Olivares, J. W. Simpson-Porco, M. Kazerani, and D. Saez, "A novel distributed control strategy for optimal dispatch of isolated microgrids considering congestion," IEEE Trans. Smart Grid, vol. 10 , no. 6, pp. 6595-6606, Nov. 2019.

[8] J. S. Gomez, D. Saez, J. W. Simpson-Porco, and R. Cardenas, "Distributed predictive control for frequency and voltage regulation in microgrids," IEEE Trans. Smart Grid, vol. 11, no. 2, pp. 1319-1329, Mar. 2020.

[9] V. Nasirian, F. L. Lewis, and A. Davoudi, "Distributed optimal dispatch for DC distribution networks," in 2015 IEEE 1st Int. Conf. Direct Curr. Microgrids, ICDCM 2015. Institute of Electrical and Electronics Engineers Inc., Jul. 2015, pp. 97-101.

[10] M. Zaery, P. Wang, W. Wang, and D. Xu, "Distributed Global Economical Load Sharing for a Cluster of DC Microgrids," IEEE Trans. Power Syst., vol. 35, no. 5, pp. 3410-3420, Sep. 2020.

[11] S. Moayedi and A. Davoudi, "Unifying Distributed Dynamic Optimization and Control of Islanded DC Microgrids," in IEEE Trans. Power Electron., vol. 32, no. 3. Institute of Electrical and Electronics Engineers Inc., Mar. 2017, pp. 2329-2346.

[12] R. Babazadeh-Dizaji and M. Hamzeh, "Distributed Hierarchical Control for Optimal Power Dispatch in Multiple DC Microgrids," IEEE Syst. J., vol. 14, no. 1, pp. 1015-1023, Mar. 2020.

[13] A. Navas F., J. S. Gómez, J. Llanos, E. Rute, D. Sáez, and M. Sumner, "Distributed predictive control strategy for frequency restoration of microgrids considering optimal dispatch," IEEE Trans. Smart Grid, pp. 1-1, Jan. 2021

[14] G. Chen and Z. Guo, "Distributed secondary and optimal active power sharing control for islanded microgrids with communication delays," IEEE Trans. Smart Grid, vol. 10, no. 2, pp. 2002-2014, Mar. 2017.

[15] F. Bullo, Lectures on network systems, 1st ed. Kindle Direct Publishing, 2020, with contributions by J. Cortes, F. Dorfler, and S. Martinez.

[16] E. F. Camacho and C. Bordons, "Constrained Model Predictive Control," in Model Predict. Control, 2nd ed., ser. Advanced Textbooks in Control and Signal Processing. London: Springer London, 2007, no. Dec., ch. 7 , pp. 177-216.

[17] E. Espina, C. Burgos-Mellado, J. S. Gomez, J. Llanos, E. Rute, F. Alex Navas, M. Martinez-Gomez, R. Cardenas, and D. Sacz, "Experimental Hybrid AC/DC-Microgrid Prototype for Laboratory Research," in 2020 22nd Eur. Conf. Power Electron. Appl. EPE 2020 ECCE Eur. Institute of Electrical and Electronics Engineers Inc., Sep. 2020.

[18] C. Ahumada, R. Cárdenas, D. Sáez, and J. M. Guerrero, "Secondary Control Strategies for Frequency Restoration in Islanded Microgrids With Consideration of Communication Delays," IEEE Trans. Smart Grid, vol. 7, no. 3, pp. 1430-1441, May 2016. 